\title{
Incontinência Urinária: Prevalência e Fatores de Risco em Mulheres em uma Unidade Básica de Saúde
}

\author{
Urinary Incontinence: Prevalence and Risk Factors in Women at a Basic Health Unit \\ Incontinencia Urinaria: Prevalencia y Factores de Riesgo en Mujeres en una \\ Unidad Básica de Salud
}

\author{
Claudia Daniella Avelino Vasconcelos Benício ${ }^{1}$, Maria Helena Barros Araújo Luz², \\ Maria Helena Baena de Moraes Lopes ${ }^{3}$, Nalma Alexandra Rocha de Carvalho ${ }^{4}$
}

\begin{abstract}
RESUMO
A incontinência urinária é definida pela International Continence Society como uma queixa de qualquer perda involuntária de urina. Este estudo objetivou estimar a prevalência de incontinência urinária; identificar os fatores de risco associados à sua ocorrência, bem como traçar os perfis sociodemográfico e clínico de mulheres atendidas no Ambulatório de Ginecologia de uma Unidade Básica de Saúde de Teresina, no Piauí. Foi um estudo exploratório-descritivo, transversal, com abordagem quantitativa. Participaram da investigação 306 mulheres entre 20 e 83 anos. Utilizou-se como instrumento de investigação um formulário estruturado composto por dados sociodemográficos e clínicos. Uma análise estatística descritiva foi realizada a partir da distribuição de frequência e dos percentuais das variáveis, empregando-se as medidas de tendência central e dispersão e o teste do $\chi^{2}$ com nível de significância de 5\% para verificar as possíveis associações entre os quesitos estabelecidos nos objetivos específicos da pesquisa. A prevalência de incontinência urinária foi de 40,8\%. A maioria dos participantes apresentou incontinência urinária de estresse $(60,0 \%)$, seguida da urgeincontinência $(28,2 \%)$ e da incontinência urinária mista (12,1\%). Houve predominância de mulheres de cor parda (64,8\%), com afecções neurológicas (72,2\%), das diabéticas (63,6\%) e hipertensas (58,1\%), daquelas submetidas às cirurgias pélvicas e abdominais (66,7\%), das obesas (52,2\%) e das tabagistas (72,2\%). O estudo possibilitou conhecer a situação da incontinência urinária em mulheres assistidas na Atenção Básica, evidenciando resultados semelhantes aos existentes na literatura, contribuindo com informações relevantes e originais sobre a incontinência urinária, podendo despertar nos profissionais e gestores de saúde pública a necessidade de maior atenção para essa clientela, no sentido de prevenção e melhoria da qualidade de vida.
\end{abstract}

DESCRITORES: Incontinência urinária. Mulheres. Fatores de risco.

\section{ABSTRACT}

The International Continence Society defines urinary incontinence as a complaint of any involuntary loss of urine. This study aimed at estimating the prevalence of urinary incontinence; identifying risk factors associated with its occurrence, as well as tracing the sociodemographic and clinical profile of women attending the Gynecology Outpatient Clinic of a Basic Health Unit in Teresina, Piauí, Brazil. This was a cross-sectional, exploratory and descriptive study, with a quantitative approach. The study included 306 women aged between 20 and 83 years old. A structured form composed

\footnotetext{
${ }_{1}^{1}$ Programa de Pós-graduação e Mestre em Enfermagem da Universidade Federal do Piauí (UFPI); Docente do Instituto Camilo Filho - Teresina (PI), Brasil. Endereço para correspondência: Claudia Daniella Avelino Vasconcelos Benício - Universitário Ministro Petrônio Portela - Ininga, Bloco 12 - CEP: $64049-550$ Teresina (PI), Brasil - E-mail: cdavb2011@yahoo.com.br

2 Professora Adjunta do Departamento de Enfermagem da UFPI - Teresina (PI), Brasil.

${ }^{3}$ Professora do Departamento de Enfermagem da UNICAMP - Campinas (SP), Brasil.

4Programa Institucional de Iniciação Científica Voluntária da UFPI - Teresina (PI), Brasil.

Artigo recebido em: 25/06/2015 - Aceito para publicação em: 05/02/2016
} 
of sociodemographic and clinical data was used as a research tool. A descriptive statistical analysis was conducted, based on the frequency distribution and percentages of variables, using measures of central tendency and dispersion, the $\chi^{2}$ test with a $5 \%$ significance level to determine possible associations between the issues established in the specific research objectives. The prevalence of urinary incontinence was of 40.8\%. Most participants presented stress urinary incontinence (60.0\%), followed by urge incontinence (28.2\%), and mixed urinary incontinence (12.1\%). Mulatto women predominated (64.8\%), women with neurological disorders (72.2\%), diabetes (63.6\%) and hypertension (58.1\%), who underwent pelvic and abdominal surgeries (66.7\%), obese (52.2\%) and smokers (72.2\%). The study enabled knowing the situation of urinary incontinence in women seen in the Primary Care, thus showing similar results to those in literature, contributing with relevant and original information about the urinary incontinence that can awaken in public health professionals and managers the need of greater attention to this clientele, regarding prevention and quality of life improvement.

DESCRIPTORS: Urinary incontinence. Women. Risk factors.

\section{RESUMEN}

La incontinencia urinaria se define por la International Continence Society como la denuncia de cualquier pérdida involuntaria de orina. Eso estudio objetivó estimar la prevalencia de la incontinencia urinaria; identificar los factores de riesgo asociados a la suya aparición, así como trazar los perfiles sociodemográficos y clínicos de las mujeres que asisten a la consulta externa de Ginecología de una Unidad Básica de Salud de Teresina, Piauí, Brasil. Fue un estudio exploratoriodescriptivo, trasversal, con un enfoque cuantitativo. El estudio incluyó a 306 mujeres de 20 años a de 83 años. Se utilizó como una herramienta de investigación una forma estructurada integrada por los datos sociodemográficos y clínicos. Un análisis estadístico descriptivo basado en la distribución de las frecuencias y porcentajes de las variables, utilizándose medidas de tendencia central y de dispersión y la prueba $\chi^{2}$ con un nivel de significación del 5\% para determinar posibles asociaciones entre las preguntas establecidas en los objetivos específicos de la investigación. La prevalencia de la incontinencia urinaria fue del 40,8\%. La mayor parte de los participantes ha presentado incontinencia urinaria de estrés (60,0\%), seguidos de la incontinencia de urgencia (28,2\%) y de la incontinencia urinaria mixta (12,1\%). Mulatas predominaron (64,8\%), las mujeres con enfermedades neurológicas (72,2\%), la diabetes (63,6\%) y la hipertensión $(58,1 \%)$, las que se sometieron a cirugía pélvica y abdominal $(66,7 \%)$, las obesas (52,2\%) y las fumadoras (72,2\%). El estudio ha permitido conocer el estado de la incontinencia urinaria en mujeres atendidas en la Atención Primaria, lo que muestra resultados similares a los encontrados en la literatura, aportando información relevante y única acerca de la incontinencia urinaria y puede despertar en los profesionales y administradores de salud pública la necesidad de una mayor atención a esta clientela en el sentido de la prevención y mejora de la calidad de vida.

DESCRIPTORES: Incontinencia urinaria. Mujeres. Factores de riesgo.

\section{INTRODUÇÃO}

A incontinência urinária (IU) é um desvio de saúde que atinge grande parte da população, especialmente o sexo feminino e com o avançar da idade. Essa enfermidade apresenta crescente prevalência em todo o mundo, despertando a preocupação e o interesse de pesquisadores, mediante as significativas alterações que provoca na vida das pessoas, as quais comprometem o seu cotidiano nos aspectos físico, psicossocial, econômico, funcional e cultural.

A queixa de qualquer perda involuntária de urina ${ }^{1}$ é a definição fornecida para a IU, que é reforçada e considerada como um sinal ou sintoma relacionado ao aparelho urinário $^{2}$, e pode provocar problemas fisiopatológicos e financeiros, além de comprometer a autoestima das pessoas, assim como as suas atividades sociais, habilidades e autonomia ${ }^{3,4}$.
A prevalência da $\mathrm{IU}$ em todo o mundo varia entre 25 e $45 \%$. No entanto, outros estudos evidenciaram a prevalência média de IU entre $27,6 \%$ em mulheres e 10,5\% em homens. Ela está presente em aproximadamente $20 \mathrm{a}$ $23 \%$ das mulheres na faixa etária dos 30 aos 39 anos, crescendo para 25 a 30\%, entre 40 e 49 anos, mantendo-se até entre 75 e 89 anos, quando ocorre um novo aumento para 30 a 35\%. Após os 90 anos, a prevalência alcança $35 \%$, podendo atingir até $50 \%$ das mulheres em alguma fase de suas vidas ${ }^{6,7}$.

Um estudo recente que teve como sua população 168 mulheres cadastradas no Sistema de Informação da Atenção Básica relativo ao Programa de Hipertensão e Diabetes, a prevalência de IU encontrada foi bastante elevada $(61,0 \%)$ e interferiu negativamente na vida das mulheres. Evidência que merece destaque, considerando-se a possibilidade de aumento da IU ao longo dos anos, uma 
vez que, mesmo sendo um estudo isolado, demonstrou um valor de prevalência maior e que não se insere na prevalência média exposta anteriormente ${ }^{8}$.

Nos Estados Unidos da América, aproximadamente 13 milhões de adultos já vivenciaram algum episódio de IU e, dentre estes, 11 milhões são mulheres, cerca de 85\% ${ }^{9,10}$. No Brasil, estudos epidemiológicos sobre a IU ainda são escassos, destacando-se as pesquisas publicadas por profissionais médicos, os quais utilizam métodos para o esclarecimento de diagnósticos, procedimentos terapêuticos e cirúrgicos, sendo menos frequentes investigações que abordem a prevalência e incidência da IU ${ }^{11,12}$.

Assim, torna-se preocupante a situação vigente, em que uma parcela de profissionais negligencia a IU e, muitas vezes, desconsidera o fato de que possivelmente mulheres adultas jovens sejam também portadoras desta condição, omitindo-se a investigar e a oferecer oportunidades para se expressarem e receberem orientações e encaminhamentos necessários à condução de tratamentos e cuidados adequados.

Nesse sentido, este trabalho objetivou traçar os perfis sociodemográfico e clínico de mulheres atendidas no Ambulatório de Ginecologia de uma Unidade Básica de Saúde (UBS) de Teresina, Piauí, bem como estimar a prevalência de IU e identificar os fatores de risco associados à sua ocorrência.

\section{METODOLOGIA}

Estudo de natureza exploratória-descritiva, de corte transversal, com abordagem quantitativa, oriundo da dissertação de Mestrado de C.D.A.V.B., realizado no município de Teresina, capital do estado do Piauí, na Unidade Integrada de Saúde do Parque Piauí.

A população do estudo foi constituída por 306 mulheres que demandaram espontaneamente consulta ginecológica na UBS, cuja amostra foi determinada pelos seguintes critérios de inclusão: mulheres com idade a partir de 20 anos que fossem capazes de responder verbalmente aos questionamentos do formulário para entrevista e que concordassem em participar da pesquisa.

A idade preestabelecida teve como propósito a possibilidade de incluir na pesquisa o maior número de mulheres possível com o desvio de saúde estudado, além de verificar a ocorrência da IU a partir desta idade. Uma vez que estudos epidemiológicos têm evidenciado que a prevalência desta enfermidade em mulheres a partir dos 30 anos varia entre 20 e $23 \%$ e considerando a idade desde os 20 anos para a inclusão das mulheres neste estudo, estimou-se para a obtenção da amostra uma prevalência presumida de 15\% de IU nas mulheres cadastradas na Coordenadoria de Região de Saúde sul urbana da Estratégia Saúde da Família. A margem de erro utilizada foi de 5\% e o nível de confiança de 95\%.

A amostragem foi do tipo intencional, selecionada no local do atendimento à medida em que as mulheres que estavam na sala de espera para consulta eram informadas sobre a pesquisa e demonstravam interesse em participar dela.

Os resultados foram processados no programa Statistical Package for the Social Sciences (SPSS 18.0 for Windows) e analisados descritivamente, medindo-se a taxa de associação entre as variáveis do estudo.

Os dados foram consolidados em um banco de dados utilizando as técnicas de estatísticas descritivas (frequência absoluta e percentual), e as medidas de tendências central (média, moda) e de dispersão (desvio padrão) foram apresentadas em forma de tabela.

Realizou-se análise estatística descritiva e exploratória a partir dos percentuais das categorias de respostas das variáveis. O uso do teste do $\chi^{2}$ com nível de significância a 5\% foi empregado para verificar as possíveis associações entre os quesitos estabelecidos nos objetivos específicos da pesquisa.

O trabalho obedeceu aos aspectos éticos e legais conforme a Resolução 466/12 do Conselho Nacional de Saúde (CNS), sendo iniciado após aprovação da Coordenação de Assuntos Assistenciais da Fundação Municipal de Saúde (FMS), bem como do Comitê de Ética em Pesquisa (CEP) da Universidade Federal do Piauí (UFPI), sob o Certificado de Apresentação para Apreciação Ética (CAAE) 0368.0.045.000-10.

\section{RESULTADOS E DISCUSSÃO}

Observou-se que, das 306 mulheres participantes do estudo, $40,8 \%$ (125) eram incontinentes, sendo que 30,4\% (38), $53,6 \%(67)$ e $16,0 \%$ (20) estavam distribuídas nas faixas etárias dos 20 a 39, 40 a 59 e 60 a 83 anos, respectivamente. Um total de 29,6\% (37) tinha o ensino médio completo; $51,2 \%$ (64) eram casadas; 40\% (50) eram naturais de Teresina; 64,8\% (81) eram pardas; 55,2\% (69) conviviam com quatro a seis pessoas na família e 36\% (45) inseriam-se nas diversas ocupações. 44,8\% (56) e 55,2\% (69) possuíam, respectivamente, renda mensal individual e familiar entre um e dois salários-mínimos (Tabela 1). 
Tabela 1. Perfil sociodemográfico das mulheres participantes do estudo ( $n=306)$, Teresina (PI), 2011.

\begin{tabular}{|c|c|c|c|c|}
\hline \multirow[t]{2}{*}{ Variáveis } & $\begin{array}{c}\text { Com incontinência } \\
\text { urinária }\end{array}$ & $\begin{array}{c}\text { Sem incontinência } \\
\text { urinária }\end{array}$ & Total & \multirow{2}{*}{$\begin{array}{c}\text { Valor } \\
\mathrm{p}\end{array}$} \\
\hline & n (\%) & n (\%) & $(n / 100 \%)$ & \\
\hline \multicolumn{5}{|l|}{ Faixa etária (anos) } \\
\hline 20 a 39 & $38(30,4)$ & $114(63,0)$ & $152(49,7)$ & \multirow{4}{*}{$-<0,001$} \\
\hline 40 a 59 & $67(53,6)$ & $55(30,4)$ & $122(40,0)$ & \\
\hline 60 a 83 & $20(16,0)$ & $12(6,6)$ & $32(10,5)$ & \\
\hline \multirow[t]{2}{*}{ Total } & $125(100,0)$ & $181(100,0)$ & $306(100,0)$ & \\
\hline & $\begin{array}{c}\text { Mediana } \\
\text { Média } \pm D P) \\
26(41,1 \pm 14,3)\end{array}$ & $\begin{array}{c}\text { IC95\% } \\
39,5-42,7\end{array}$ & $\begin{array}{l}\text { Mínimo- } \\
\text { Máximo } \\
20-83\end{array}$ & $\begin{array}{c}\text { Valor } \\
p\end{array}$ \\
\hline \multicolumn{5}{|l|}{ Escolaridade } \\
\hline Não alfabetizado & $9(7,2)$ & $9(5,0)$ & $18(5,9)$ & \multirow{8}{*}{0,160} \\
\hline Fundamental incompleto & $30(24,0)$ & $34(19,0)$ & $64(20,9)$ & \\
\hline Fundamental completo & $12(9,6)$ & $17(9,4)$ & $29(9,5)$ & \\
\hline Médio incompleto & $25(20,0)$ & $22(12,1)$ & $47(15,4)$ & \\
\hline Médio completo & $37(29,6)$ & $69(38,1)$ & $106(34,6)$ & \\
\hline Superior incompleto & $6(4,8)$ & $19(10,5)$ & $25(8,1)$ & \\
\hline Superior completo & $6(4,8)$ & $11(6,0)$ & $17(5,6)$ & \\
\hline Total & $125(100,0)$ & $181(100,0)$ & $306(100,0)$ & \\
\hline \multicolumn{5}{|l|}{ Situação conjugal } \\
\hline Casada & $64(51,2)$ & $83(45,9)$ & $147(48,0)$ & \multirow{6}{*}{0,103} \\
\hline União estável & $8(6,4)$ & $18(9,9)$ & $26(8,4)$ & \\
\hline Solteira & $28(22,4)$ & $59(32,6)$ & $87(28,4)$ & \\
\hline Separada & $13(10,4)$ & $11(6,0)$ & $24(7,8)$ & \\
\hline Viúva & $12(9,6)$ & $10(5,5)$ & $22(7,4)$ & \\
\hline Total & $125(100,0)$ & $181(100,0)$ & $306(100,0)$ & \\
\hline \multicolumn{5}{|l|}{ Naturalidade } \\
\hline Teresina & $50(40,0)$ & $89(49,2)$ & $139(45,4)$ & \multirow{4}{*}{0,236} \\
\hline Interior do Piauí & $43(34,4)$ & $57(31,5)$ & $100(32,7)$ & \\
\hline Outros estados & $32(25,6)$ & $35(19,3)$ & $67(21,9)$ & \\
\hline Total & $125(100,0)$ & $181(100,0)$ & $306(100,0)$ & \\
\hline \multicolumn{5}{|l|}{ Cor } \\
\hline Parda & $81(64,8)$ & $119(65,7)$ & $200(65,4)$ & \multirow{4}{*}{0,923} \\
\hline Branca & $24(19,2)$ & $36(19,9)$ & $60(19,6)$ & \\
\hline Negra & $20(16,0)$ & $26(14,4)$ & $46(15,0)$ & \\
\hline Total & $125(100,0)$ & $181(100,0)$ & $306(100,0)$ & \\
\hline \multicolumn{5}{|c|}{ Número de pessoas na família } \\
\hline 1 a 3 & $40(32,0)$ & $61(33,7)$ & $101(33,0)$ & \multirow{5}{*}{0,895} \\
\hline 4 a 6 & $69(55,2)$ & $94(51,9)$ & $163(53,3)$ & \\
\hline 7 a 10 & $14(11,2)$ & $24(13,3)$ & $38(12,4)$ & \\
\hline 11 e mais & $2(1,6)$ & $2(1,1)$ & $4(1,3)$ & \\
\hline Total & $125(100,0)$ & $18(100,0)$ & $306(100,0)$ & \\
\hline
\end{tabular}


Tabela 1. Continuação.

\begin{tabular}{|c|c|c|c|c|}
\hline \multirow[t]{2}{*}{ Variáveis } & $\begin{array}{c}\text { Com incontinência } \\
\text { urinária }\end{array}$ & $\begin{array}{c}\text { Sem incontinência } \\
\text { urinária }\end{array}$ & Total & \multirow{2}{*}{$\begin{array}{c}\text { Valor } \\
\mathrm{p}\end{array}$} \\
\hline & n (\%) & n (\%) & $(n / 100 \%)$ & \\
\hline \multicolumn{5}{|l|}{ Ocupação } \\
\hline Autônoma & $29(23,2)$ & $29(16,0)$ & $58(19,0)$ & \multirow{7}{*}{0,178} \\
\hline Do lar & $33(26,4)$ & $45(24,9)$ & $78(25,5)$ & \\
\hline Aposentada & $7(5,6)$ & $7(3,9)$ & $14(4,6)$ & \\
\hline Beneficiária & $9(7,2)$ & $23(12,7)$ & $32(10,4)$ & \\
\hline Estudante & $2(1,6)$ & $10(5,5)$ & $12(3,9)$ & \\
\hline Outra & $45(36,0)$ & $67(37,0)$ & $112(36,6)$ & \\
\hline Total & $125(100,0)$ & $181(100,0)$ & $306(100,0)$ & \\
\hline \multicolumn{5}{|c|}{ Renda mensal individual (salário-mínimo) } \\
\hline$<1$ & $41(32,8)$ & $54(29,8)$ & $95(31,0)$ & \multirow{6}{*}{0,888} \\
\hline 1 a 2 & $56(44,8)$ & $82(45,3)$ & $138(45,1)$ & \\
\hline 3 a 5 & $8(6,4)$ & $9(5,0)$ & $17(5,6)$ & \\
\hline 6 e mais & $2(1,6)$ & $3(1,7)$ & $5(1,6)$ & \\
\hline Sem renda & $18(14,4)$ & $33(18,2)$ & $51(16,7)$ & \\
\hline Total & $125(100,0)$ & $181(100,0)$ & $306(100,0)$ & \\
\hline \multicolumn{5}{|c|}{ Renda familiar (salário-mínimo) } \\
\hline$<1$ & $10(8,0)$ & $15(8,3)$ & $25(8,1)$ & \multirow{5}{*}{0,458} \\
\hline 1 a 2 & $69(55,2)$ & $101(55,8)$ & $170(55,6)$ & \\
\hline 3 a 5 & $35(28,0)$ & $57(31,5)$ & $92(30,1)$ & \\
\hline 6 e mais & $11(8,8)$ & $8(4,4)$ & $19(6,2)$ & \\
\hline Total & $125(100,0)$ & $181(100,0)$ & $306(100,0)$ & \\
\hline
\end{tabular}

*O valor p foi obtido pelo teste do $\chi^{2}$ de Pearson; DP: desvio padrão; nível de significância fixado em <0,05.

Verificou-se ainda que ao realizar a correlação pelo teste do $\chi^{2}$, entre a ocorrência de IU e as variáveis avaliadas no perfil sociodemográfico, apenas a faixa etária apresentou valor p com significância estatística $(<0,001)$, desta forma confirmando a associação entre esta variável e a IU.

Pesquisas apontam que mulheres a partir dos 45 anos apresentam algum tipo de IU, aumentando o seu percentual a partir dos 50 anos $^{13,14}$. Esses achados atribuídos ao aumento da idade, portanto ao envelhecimento, podem ser explicados pelo desgaste natural das fibras musculares, que pode provocar hipotrofia ou substituição das fibras por células do tecido conjuntivo e adiposo. Assim, ocorre um comprometimento da musculatura do assoalho pélvico, reduzindo a capacidade de colaborar para a ação da continência ${ }^{15,16}$.

O tipo de IU que prevaleceu no estudo foi a IU de estresse (IUE) com 60,0\% (75); em segundo lugar, a urgeincontinência com 28,2\% (35) mulheres; e em terceiro, a IU mista (IUM) com 12,1\% (15). Tais dados também corroboram com uma recente pesquisa que encontrou resultados com classificação de IU na mesma ordem ${ }^{17}$.

Durante o período da menopausa, a IUE representa a principal queixa de IU entre as mulheres, seguida pela urgeincontinência ${ }^{18}$. Em nosso meio, durante um importante estudo com mulheres entre 35 e 81 anos, foram identificados percentuais para IUE, IU e IUM de 30,$7 ; 14,2$ e 10,0 , respectivamente ${ }^{19}$.

A prevalência de IU encontrada nesta pesquisa teve valor de 40,8\%. Esse é um percentual considerado elevado, porém dentro dos parâmetros encontrados em outros estudos realizados e publicados na literatura, dentre os quais citam-se totais oscilando entre 12 e $55 \%$ em mulheres ${ }^{20,21}$. 
Em um estudo brasileiro, encontrou-se uma prevalência de $32,9 \%$ em mulheres e $6,2 \%$ em homens, considerando adultos com idade igual ou superior a 18 anos e $\mathrm{n}=51.922$. Já em outro, houve uma prevalência de 23,50\% para mulheres com idade a partir de 16 anos $(n=332)^{23}$.

Com relação aos fatores de risco, embora já tenha sido evidenciada em vários estudos a associação entre a prevalência de IU e fatores de risco como doenças neurológicas, diabetes, hipertensão, doença pulmonar obstrutiva crônica (DPOC), tabagismo, consumo de cafeína, cirurgias pélvicas e abdominais, uso de medicação anti-hipertensiva, obesidade e constipação ${ }^{4,11,13,24-27}$, neste estudo não foi observada associação entre estes dados clínicos e a IU, por meio da aplicação do teste de $\chi^{2}$. No entanto, conforme a Tabela 2 , considerou-se que os achados referentes a estas variáveis constituíram importantes fatores que podem predispor a ocorrência da IU, pois os seus percentuais foram mais elevados nas mulheres incontinentes, o que mostra uma tendência de associação entre as referidas condições. Vale ressaltar que quanto ao uso dos antidiabéticos como fator de risco para a ocorrência de IU, além de não ter sido verificada correlação estatística com a IU, não foram encontrados também na literatura estudos relacionados (Tabela 2).

Entretanto, ao se avaliar a variável 'número de gestações' na Tabela 3, notou-se correlação significativa desta com a prevalência de IU ( $p=0,02)$. Esse achado é reforçado por relevante pesquisa que afirma que os episódios obstétricos são considerados os fatores de risco mais importantes para o desenvolvimento da IU, sendo o trauma do períneo durante o parto, o evento principal para a sua ocorrência ${ }^{28}$ (Tabela 3 ).

O estudo de 336 mulheres do Programa Saúde da Família de Dourados (Mato Grosso do Sul) confirmou,estatisticamente, em seus resultados, que o número de gestações e a paridade apresentam correlação com a IU, portanto comprometendo gravemente a qualidade de vida ${ }^{29}$. Da mesma forma, em outros estudos, apresentou-se que a gravidez, o grande número de partos e o parto vaginal contribuem para o desenvolvimento da IUE ${ }^{30,31}$, enquanto as mulheres nulíparas são menos comprometidas quanto ao aparecimento da perda de urina ${ }^{28}$.

Tabela 2. Associação entre a prevalência de incontinência urinária e dos fatores de risco estudados $(\mathrm{n}=306)$, Teresina (PI), 2011.

\begin{tabular}{|c|c|c|c|c|}
\hline \multirow[b]{2}{*}{ Fatores de risco estudados } & \multicolumn{4}{|c|}{ Prevalência de incontinência urinária } \\
\hline & $\begin{array}{c}\text { Sim } \\
\text { n (\%) }\end{array}$ & $\begin{array}{c}\text { Não } \\
\text { n (\%) }\end{array}$ & $\begin{array}{l}\text { Total } \\
\text { (n) }\end{array}$ & $\begin{array}{c}\text { Valor } \\
p^{*}\end{array}$ \\
\hline \multicolumn{5}{|l|}{ Enfermidades associadas } \\
\hline Doenças neurológicas & $13(72,2)$ & $5(27,8)$ & 18 & \multirow{4}{*}{0,20} \\
\hline Diabetes & $14(63,6)$ & $8(36,4)$ & 22 & \\
\hline Hipertensão & $43(58,1)$ & $31(41,9)$ & 74 & \\
\hline Doença pulmonar obstrutiva crônica & $4(44,4)$ & $5(55,6)$ & 9 & \\
\hline \multicolumn{5}{|l|}{ Hábitos } \\
\hline Tabagismo & $26(72,2)$ & $10(27,8)$ & & \multirow{2}{*}{36} \\
\hline Cafeína & $46(51,1)$ & $44(48,9)$ & 90 & \\
\hline \multicolumn{5}{|l|}{ Cirurgias } \\
\hline Pélvicas e abdominais & $16(66,7)$ & $8(33,3)$ & 24 & \\
\hline Abdominais & $20(57,1)$ & $15(42,9)$ & 35 & \\
\hline Pélvicas & $80(49,4)$ & $82(50,6)$ & 162 & \\
\hline \multicolumn{5}{|l|}{ Medicações } \\
\hline Diversas & $57(52,8)$ & $51(47,2)$ & 108 & \\
\hline Antidiabética & $13(59,1)$ & $9(40,9)$ & 22 & \\
\hline Anti-hipertensiva & $40(57,1)$ & $30(42,9)$ & 70 & \\
\hline \multicolumn{5}{|l|}{ Condições nutricional e de eliminação } \\
\hline Obesidade & $24(52,2)$ & $22(47,8)$ & 46 & \\
\hline Constipação & $50(51,0)$ & $48(49,0)$ & 98 & \\
\hline
\end{tabular}

*O valor $p$ foi obtido pelo teste de $\chi^{2}$. Nível de significância fixado em $<0,05$. 
Tabela 3. Análise da diferença entre as variáveis e os grupos de quem apresenta e não apresenta incontinência urinária ( $n=306)$, Teresina (PI), 2011.

\begin{tabular}{|c|c|c|c|}
\hline \multirow[b]{2}{*}{ Variáveis } & \multicolumn{3}{|c|}{ Prevalência de incontinência urinária } \\
\hline & $\begin{array}{l}\text { Sim } \\
\text { média }\end{array}$ & $\begin{array}{l}\text { Não } \\
\text { média }\end{array}$ & $\begin{array}{c}\text { Valor } \\
p^{*}\end{array}$ \\
\hline Número de gestações & 4,0 & 3,3 & 0,02 \\
\hline Número de abortos & 1,5 & 1,6 & 0,44 \\
\hline Número de partos normais & 2,2 & 2,2 & 0,06 \\
\hline Número de partos cesarianos & 1,6 & 1,5 & 0,33 \\
\hline
\end{tabular}

*O valor $p$ foi obtido pelo teste de Mann-Whitney. Nível de significância fixado em <0,05.

Tais resultados recebem mais ênfase pelos achados de que a IU e fecal têm início durante a gestação e que a história familiar positiva e o parto vaginal são fatores de risco independentes para a persistência dos sintomas de IU e fecal no pós-parto imediato ${ }^{32}$.

A literatura sobre IU vem sendo ampliada em todo o mundo, demonstrando que o interesse pela investigação sobre esta temática cresce, o que confirma um maior estímulo por parte dos profissionais de saúde em buscarem mais informações referentes aos questionamentos que ainda não foram elucidados sobre o assunto, ou ratificarem hipóteses sugeridas/indagadas. No entanto, o que se observa é que estes estudos têm se limitado a pesquisas envolvendo grupos específicos como o de mulheres, principalmente nos períodos gestacional e puerperal, idosos e pacientes ambulatoriais e institucionalizados em hospitais ou clínicas especializadas em urologia, ginecologia e uroginecologia, em que a demanda de pessoas incontinentes é significativa.

No Brasil, a literatura já foi mais escassa, porém ainda demanda trabalhos mais impactantes que provoquem o despertar dos gestores de saúde para o problema da IU, seus fatores de risco e suas consequências que se tornam mais evidentes, principalmente e infelizmente, apenas para grupos de profissionais que estudam especificamente Estomaterapia, saúde da mulher, do homem e do idoso.

\section{CONSIDERAÇÕES FINAIS}

Diante do contexto vivenciado, pode-se ressaltar que a falta de sensibilização, de conhecimento e de capacitação de alguns profissionais de saúde para lidar com a IU pode torná-los inaptos para assistir adequadamente as mulheres com IU. Nesse contexto, a Enfermagem é inserida e destacada como uma categoria que ainda pouco contribui para o cuidado da pessoa com IU, embora existam possibilidades de especialização relacionada à prática assistencial junto à clientela incontinente.

Desse modo, tornam-se necessários e urgentes o treinamento e o desenvolvimento das estratégias de capacitação por parte dos gestores de saúde na tentativa de sensibilizar os profissionais de saúde a promoverem o cuidado às mulheres com IU, assistindo-as desde a prevenção, como no tratamento para a cura da IU.

Acredita-se que a Enfermagem, mais especificamente os enfermeiros que atuam em programas voltados para a assistência da saúde da mulher e os que estão inseridos nas Equipes de Saúde da Família, são os que provavelmente teriam mais oportunidades de promover o cuidado direcionado à prevenção da IU e promoção da continência, em função da possibilidade de realizar consultas individuais a essas mulheres e fornecer palestras educativas. Assim, julga-se importante a inserção desta temática desde a graduação na área de Enfermagem, para que, enquanto profissionais enfermeiros, seja possível contribuir de maneira significativa na prevenção e melhoria da continência nas mulheres que experimentam este problema.

Finalmente, supõe-se que este estudo poderá além de fornecer dados e informações originais sobre a IU em mulheres, despertar nos profissionais e gestores de saúde pública a necessidade de voltarem à atenção para pessoas com IU. 


\section{REFERÊNCIAS}

1. Haylen BT, Ridder D, Freeman RM, Swift SE, Berghmans B, Lee J, et al. Floor Dysfunction. Standardization and Terminology Committees IUGA and ICS, Joint IUGA / ICS Working Group on Female Terminology. Neurourol Urodyn. 2010;29(1):4-20.

2. Abrams P, Andersson KE, Artibani W, Brubaker L, Cardoso $L$, Castro D, et al. Recommendations of the International Scientific Committee: evaluation and treatment of urinary incontinence, pelvic organ prolapse and faecal incontinence. In: 2nd International Consultation on Incontinence; 2001, Paris, France. ICUD/ICS; 2002. p. 1079-96.

3. Organização Mundial da Saúde. Centro Colaborador da OMS para a Classificação de Doenças em Português CBCD. Classificação Estatística Internacional de Doenças e Problemas Relacionados à Saúde - CID 10. [acesso em 15 dez. 2011]. Disponível em: <http://www.datasus.gov.br/ cid10/v2008/cid10.htm>

4. Azevedo GR, Declair V, Silva S. Enfermagem atual em cursos: estomaterapia, incontinências urinária e anal, Programa de saúde da família - PSF, Feridas, Atualização de enfermagem, em dermatologia. Petrópolis: bbEPUB; 2005.

5. Higa R, Lopes MH, Reis MJ. Fatores de risco para incontinência urinária na mulher. Rev Esc Enferm USP. 2008;42(1):187-92.

6. Buckley BS, Lapitan MC, Epidemiology Committee of the Fourth International Consultation on Incontinence. Paris, 2008. Prevalence of urinary incontinence in men, women, and children - current evidence: findings of the Fourth International Consultation on Incontinence. Urology. 2010;76(2):265-70.

7. Minassian VA, Drutz HP, Al-Badr A. Urinary incontinence as a worldwide problem. Int J Gynaecol Obstet. 2003;82(3):327-38.

8. Fonseca ES, Camargo AL, Castro RA, Sartori MG, Fonseca MC, Lima GR, et al. Validação do questionário de qualidade de vida (King's Health Questionnaire) em mulheres brasileiras com incontinência urinária. Rev Bras Ginecol Obstet. 2005;27(5):235-42.

9. Menezes GM, Pinto FJ, Silva FA, Castro MD, Medeiros CR. Queixa de perda urinária: um problema silente pelas mulheres. Rev Gaúcha Enferm. 2012;33(1):100-8.

10. Lopes $M H$, Higa R. Restrições causadas pela incontinência urinária à vida da mulher. Rev Esc Enferm USP. 2006;40(1):34-41.

11. Santos VL, Cesaretti IR. Assistência em estomaterapia: cuidando do ostomizado. 1. ed. São Paulo: Atheneu; 2005.

12. Tamanini JT, Lebrão ML, Duarte $Y A$, Santos JL, Laurenti R. Analysis of the prevalence of and factors associated with urinary incontinence among elderly people in the Municipality of São Paulo, Brazil: SABE Study (Health, Wellbeing and Aging). Cad Saúde Pública [online]. 2009;25(8):1756-62.

13. Guarisi T, Pinto Neto AM, Osis MJ, Pedro AO, Paiva LH, Faúndes A. Incontinência urinária entre mulheres climatéricas brasileiras: inquérito domiciliar. Rev Saúde Pública. 2001;35(5):428-35.

14. Higa R, Lopes MH. Fatores associados com a incontinência urinária na mulher. Rev Bras Enferm. 2005;58(4):422-8.

15. Markland AD, Richter HE, Fwu CW, Eggers P, Kusek JW. Prevalence and trends of urinary incontinence in adults in the United States, 2001 to 2008. J Urol. 2011;186(2):589-93.

16. Baracho E. Fisioterapia aplicada à obstetrícia, uroginecologia e aspectos de mastologia. 4. ed. Rio de Janeiro: Guanabara Koogan; 2007.
17. Goldstein SR, Johnson S, Watts NB, Ciaccia AV, Elmerick $D$, Muram D. Incidence of urinary incontinence in postmenopausal women treated with raloxifene or estrogen. Menopause. 2005;12(12):160-4.

18. Faria CA, Menezes AM, Rodrigues AO, Ferreira AL, Bolsas CN. Incontinência urinária e noctúria: prevalência e impacto sobre qualidade de vida em idosas numa Unidade Básica de Saúde. Rev Bras Geriatr Gerontol. 2014;17(1):17-25.

19. Bortolotti A, Bernardini B, Colli E, Di Benedetto P, Giocoli Nacci $G$, Landoni $M$, et al. Prevalence and risk factors for urinary incontinence in Italy. Eur Urol. 2000;37(1):30-5.

20. Guarisi T, Pinto Neto AM, Osisb MJ, Pedro AO, Paiva LH, Faúndes A. Sintomas urinários e genitais em mulheres climatéricas. J Bras Ginecol. 1998;108(4):125-30.

21. Siracusano S, Pregazzi R, d'Aloia G, Sartore A, Di Benedetto $P$, Pecorari $V$, et al. Prevalence of urinary incontinence in young and middle-aged women in an Italian urban area. Eur J Obstet Gynecol Reprod Biol. 2003;107(2):201-4.

22. Thom $\mathrm{DH}$. Variation in estimates of urinary incontinence prevalence in the community: effect of differences in definition, population characteristics and study type. J Am Geriatr Soc. 1998;46(4):473-80.

23. Santos C, Santos VL. Prevalence of Urinary incontinence in a random sample of the urban population of Pouso Alegre, Minas Gerais, Brazil. Rev Latino-am Enfermagem. 2010;18(5):[8telas].

24. Borges JB, Borges JB, Guarisi T, Camargo AC, Gollop TR, Machado RB, et al. Incontinência urinária após parto vaginal ou cesáreo. Einstein J. 2010;8(2):192-6.

25. Merola A, Zibetti M, Angrisano S, Rizzi L, Ricchi V, Artusi CA, et al. Parkinson's disease progression at 30 years: a study of subthalamic deep brain-stimulated patients. Brain. 2001;134(7):2074-84.

26. Izci Y, Topsever P, Filiz TM, Çınar ND, Uludağ C, LagroJanssen $T$. The association between diabetes mellitus and urinary incontinence in adult women. Int Urogynecol J Pelvic Floor Dysfunct. 2009;20(8):947-52.

27. Hrisanfow $E$, Hägglund D. The prevalence of urinary incontinence among women and men with chronic obstructive pulmonary disease in Sweden. J Clin Nursing. 2011;20(13-14):1895-905.

28. Stepan J, Kalis V, Novotný Z, Havir M, Chaloupka P, Rokyta Z. The impact of total abdominal and laparoscopically assisted vaginal hysterectomy on the development of urinary incontinence. Ceska Gynecol. 2006;71(5):438-45.

29. Rortveit G, Daltveit AK, Hannestad YS, Hunskaar S. Epincont Study. Urinary incontinence after vaginal delivery or cesarean section. N Engl J Med. 2003;348(10):900-7.

30. Gomes G, Silva GD. Incontinência urinária de esforço em mulheres pertencentes ao Programa de Saúde da Família de Dourados (MS). Rev Assoc Med Bras. 2010;56(6):649-54.

31. Amaro JL, Yamamoto $H$, Kawano PR, Barros G, Gameiro $\mathrm{MO}$, Agostinho AD. Clinical and quality-of-life outcomes after autologous fascial sling and tension-free vaginal tape: a prospective randomized Trial. Int Braz J Urol. 2009;35(1):60-7.

32. Kepenekci I, Keskinkilic B, Akinsu F, Cakir P, Elhan AH, Erkek $A B$, et al. Prevalence of pelvic floor disorders in the female population and the impact of age, mode of delivery, and parity. Dis Colon Rectum. 2011;54(1):85-94. 